\title{
Enumerating Lattice Paths Touching or Crossing the Diagonal at a Given Number of Lattice Points
}

\author{
Michael Z. Spivey \\ Department of Mathematics and Computer Science \\ University of Puget Sound \\ Tacoma, Washington 98416-1043 \\ USA \\ mspivey@pugetsound.edu
}

Submitted: Jan 27, 2012; Accepted: Aug 17, 2012; Published: Aug 30, 2012

Mathematics Subject Classifications: 05A15, 05A19

\begin{abstract}
We give bijective proofs that, when combined with one of the combinatorial proofs of the general ballot formula, constitute a combinatorial argument yielding the number of lattice paths from $(0,0)$ to $(n, r n)$ that touch or cross the diagonal $y=r x$ at exactly $k$ lattice points. This enumeration partitions all lattice paths from $(0,0)$ to $(n, r n)$. While the resulting formula can be derived using results from Niederhausen, the bijections and combinatorial proof are new.
\end{abstract}

Keywords: lattice path; bijection

\section{Introduction}

In this paper we consider lattice paths from $(0,0)$ to $(n, r n)$ with the following properties.

1. The paths use only unit steps right and units steps up.

2. The paths touch exactly $k$ lattice points of the line $y=r x$ after leaving $(0,0)$. The paths can cross $y=r x$ or not at these lattice points. The point $(0,0)$ is not included among these $k$ lattice points, but $(n, r n)$ is.

3. The paths may cross the line $y=r x$ any number of times at non-lattice points. 
Our main result is a combinatorial proof that the number of such paths is

$$
\frac{(r+1)^{k} r k}{(r+1) n-k}\left(\begin{array}{c}
(r+1) n-k \\
r n
\end{array}\right) .
$$

Our proof is based on two bijections; it also assumes a combinatorial proof of the general ballot formula (e.g., Goulden and Serrano [3]) as well. In addition, since every lattice path from $(0,0)$ to $(n, r n)$ is counted in one of these sets of paths for exactly one $k$, we have a combinatorial proof of the formula

$$
\sum_{k=1}^{n} \frac{(r+1)^{k} r k}{(r+1) n-k}\left(\begin{array}{c}
(r+1) n-k \\
r n
\end{array}\right)=\left(\begin{array}{c}
(r+1) n \\
n
\end{array}\right) .
$$

Alternatively, expression (1) gives the number of random walks of $r n+n$ steps using step sizes of -1 and $+r$ that return to the origin $k-1$ times before ending at the origin for the $k$ th time.

Formula (1) does not appear to be explicitly stated anywhere in the literature, although it can be derived from some results by Niederhausen [7]. He proves that the number of paths that touch at least $k$ of the lattice points (rather than exactly $k$ ) is given by $(r+1)^{k}\left(\begin{array}{c}(r+1) n-k \\ r n\end{array}\right)$. Thus the number of paths that touch exactly $k$ of the lattice points is $(r+1)^{k}\left(\begin{array}{c}(r+1) n-k \\ r n\end{array}\right)-(r+1)^{k+1}\left(\begin{array}{c}(r+1) n-(k+1) \\ r n\end{array}\right)$, which can be simplified to give the expression in formula (1). Niederhausen's proof uses Sheffer sequences, though, rather than bijections.

Other variations and special cases of formula (1) appear elsewhere. The case $r=1$ is proved by McGregor, Narayana, and Özsoyoglu [5], by Engelberg [1], and by Feller [2]. Mohanty [6] considers a restricted version of the general case in which the lattice paths are not allowed to cross the line $y=r x$. The recent survey paper by Humphreys [4] mentions some additional variations.

Our first bijection shows that paths from $(0,0)$ to $(n, r n)$ that do not touch a lattice point of $y=r x$ except at the start and finish can be partitioned into $r+1$ sets of equal size. This partition is based on how far below the line $y=r x$ each path goes when it first steps over the line. Our second bijection is between paths from $(0,0)$ to $(n, r n)$ that touch $y=r x$ exactly $k$ times after the start but do not go below it and paths from $(0,0)$ to $(n-k, r n)$ that never touch the line $y=r x$ after the start. The latter are counted by the general ballot theorem. Our two bijections and the ballot theorem together give us the formula (1).

\section{The bijections}

Let $\mathcal{L}_{r, n}$ be the set of lattice paths from $(0,0)$ to $(n, r n)$ that do not touch a lattice point on the line $y=r x$ except at $(0,0)$ and $(n, r n)$. (The paths in $\mathcal{L}_{r, n}$ can cross the line $y=r x$, just not at a lattice point.) Since unit up steps cannot cross the line $y=r x$ other than at a lattice point, for each path $p$ in $\mathcal{L}_{r, n}$ there is some $i, 0 \leqslant i \leqslant r$, such that the first time $p$ goes below the line $y=r x$ it does so with a unit right step that lands on a 
lattice point of $y=r x-i$, and from there on $p$ remains below $y=r x$ until the end. (For paths that never go below $y=r x$ we say $i=0$, and for paths that never go above $y=r x$ we say $i=r$.) Partition $\mathcal{L}_{r, n}$ into $r+1$ disjoint subsets $\mathcal{L}_{r, n, i}$, based on the value of $i$ for each path. Our first result is the following.

Theorem 1. There is an explicit bijection $\phi_{i}: \mathcal{L}_{r, n, i} \mapsto \mathcal{L}_{r, n, r}$ for each $i, 0 \leqslant i \leqslant r-1$.

Proof. Let $p \in \mathcal{L}_{r, n, i}$. By definition, there must be some step $p_{m}$ in the path $p$ such that $p_{m}$ is the first step that goes below $y=r x, p_{m}$ lands on a lattice point of $y=r x-i$, and after step $p_{m}$ the path $p$ lies completely below $y=r x$ until $(n, r n)$. Create a new path $p^{\prime}$ by rotating by $180^{\circ}$ the initial part of $p$ up through and including step $p_{m}$. This rotation maps points on the line $y=r x$ to points on the line $y=r x-i$, so after leaving $(0,0) p^{\prime}$ lies strictly below $y=r x$ through step $p_{m}^{\prime}$ and touches a lattice point of $y=r x-i$ for the first time at step $p_{m}^{\prime}$. Since $p^{\prime}$ agrees with $p$ after the $m$ th step, the rest of path $p^{\prime}$ must lie below $y=r x$ until $(n, r n)$ as well. Thus $p^{\prime} \in \mathcal{L}_{r, n, r}$. (See, for example, Figure 1.)

Also, note that each path in $\mathcal{L}_{r, n, r}$ must end with $r$ unit steps up. Thus each path in $\mathcal{L}_{r, n, r}$ must reach a lattice point on the line $y=r x-i$ at least once and therefore must do so somewhere for the first time. Thus the mapping is reversible. (For example, the path $p^{\prime} \in \mathcal{L}_{r, n, r}$ in Figure 1 touches a lattice point of $y=r x-i$ three times. The part of $p^{\prime}$ through the first time $p^{\prime}$ touches a lattice point of $y=r x-i$ is reversed to obtain a path in $\left.\mathcal{L}_{r, n, i} \cdot\right)$

The proof of Theorem 1 bears some similarity to the combinatorial proof of the general ballot theorem by Goulden and Serrano [3], in that both proofs partition paths based on the first passage time across the line $y=r x$ to a lattice point on $y=r x-i$, and both proofs feature a rotation of the initial part of the path.

Theorem 2. There is an explicit bijection between paths from $(0,0)$ to $(n, r n)$ that touch the line $y=r x$ in exactly $k$ places after $(0,0)$ but never go below it and paths from $(0,0)$ to $(n-k, r n)$ that do not touch the line $y=r x$ after $(0,0)$.

Proof. Given a path in the first group, remove, starting at the end of the path, each step in the path that touches the line $y=r x$. This produces a path in the second group. To reverse the mapping, find the last place the path touches a lattice point of $y=r x+r$. There must be such a point because the path touches $(0, r)$. Insert a unit right step at this place in the path. This creates a new path that touches the line $y=r x$ a second time. This new path must have a last place that it touches a lattice point of $y=r x+r$. Insert a unit right step at this place in the new path. Continue this process until the path touches $(n, r n)$. Since we always choose the last place a path touches a lattice point of $y=r x+r$, this is a reversal of the original mapping. See, for example, Figure 2. 


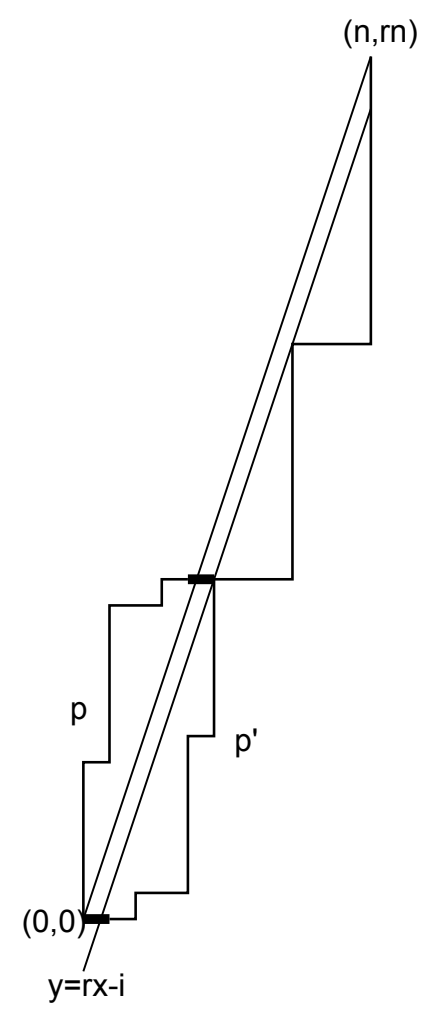

Figure 1: Bijection between paths in $\mathcal{L}_{r, n, i}$ and $\mathcal{L}_{r, n, r}$. The initial part of $p$ is rotated to obtain $p^{\prime}$.

\section{Main Result}

For our main result we need the general ballot theorem (for instance, [3]): The number of paths from $(0,0)$ to $(n, m), m>r n$, that never touch the line $y=r x$ after $(0,0)$ is given by

$$
\frac{m-r n}{m+n}\left(\begin{array}{c}
m+n \\
m
\end{array}\right)
$$

Our main result is a combinatorial proof of the following theorem.

Theorem 3. The number of lattice paths from $(0,0)$ to $(n, r n)$ that touch or cross the line $y=r x$ at exactly $k$ lattice points after $(0,0)$ but can stay above, below, or cross it in any number of other places is

$$
\frac{(r+1)^{k} r k}{(r+1) n-k}\left(\begin{array}{c}
(r+1) n-k \\
r n
\end{array}\right) .
$$

Proof. By Theorem 2 and the ballot theorem, the number of these paths that never go below $y=r x$ is

$$
\frac{r k}{(r+1) n-k}\left(\begin{array}{c}
(r+1) n-k \\
r n
\end{array}\right) \text {. }
$$




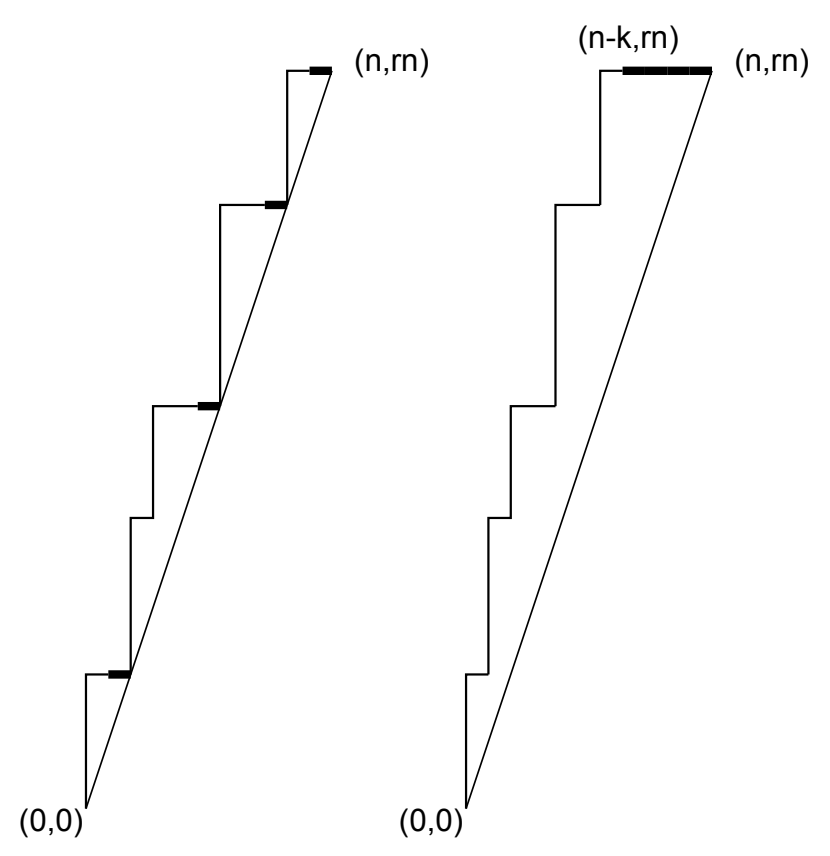

Figure 2: Bijection between paths touching $y=r x$ at $k$ places after the start, ending at $(n, r n)$, and paths touching $y=r x$ nowhere after the start, ending at $(n-k, r n)$

Given such a path $p$ that never goes below $y=r x$, divide it into $k$ segments based on the locations $p$ touches or crosses the line $y=r x$ : The first segment $p_{1}$ is from $\left(x_{0}, r x_{0}\right)=(0,0)$ to the first return at $\left(x_{1}, r x_{1}\right)$, the second segment $p_{2}$ is from $\left(x_{1}, r x_{1}\right)$ to the second return at $\left(x_{2}, r x_{2}\right)$, and so forth. Since the $j$ th segment $p_{j}$ does not touch the line $y=r x$ except at $\left(x_{j-1}, r x_{j-1}\right)$ and $\left(x_{j}, r x_{j}\right)$, applying the bijection in Theorem 1 to $p_{j}$ (via $\left.\mathcal{L}_{r, n, 0} \leftrightarrow \mathcal{L}_{r, n, r} \leftrightarrow \mathcal{L}_{r, n, i}\right)$ maps it to $r+1$ paths from $\left(x_{j-1}, r x_{j-1}\right)$ to $\left(x_{j}, r x_{j}\right)$ that do not touch a lattice point on the line $y=r x$ except at $\left(x_{j-1}, r x_{j-1}\right)$ and $\left(x_{j}, r x_{j}\right)$. This bijection can be applied to any of the $k$ segments of $p$ independently of the others, though, and so $p$ corresponds to $(r+1)^{k}$ lattice paths from $(0,0)$ to $(n, r n)$ that touch or cross the line $y=r x$ at the same $k$ lattice points after $(0,0)$ that $p$ does. Moreover, each of these $(r+1)^{k}$ correspondences is reversible, as the mapping in Theorem 1 is a bijection. Thus we have a partition of the lattice paths we are trying to count into

$$
\frac{r k}{(r+1) n-k}\left(\begin{array}{c}
(r+1) n-k \\
r n
\end{array}\right)
$$

classes, one for each path $p$ that never goes below $y=r x$, and each class has size $(r+1)^{k}$. Thus there are

$$
\frac{(r+1)^{k} r k}{(r+1) n-k}\left(\begin{array}{c}
(r+1) n-k \\
r n
\end{array}\right)
$$

total paths.

Summing up over all values of $k$ in Theorem 3 yields the total number of lattice paths from $(0,0)$ to $(n, r n)$ and thus completes a combinatorial proof of the following identity. 


\section{Corollary 1.}

$$
\sum_{k=1}^{n} \frac{(r+1)^{k} r k}{(r+1) n-k}\left(\begin{array}{c}
(r+1) n-k \\
r n
\end{array}\right)=\left(\begin{array}{c}
(r+1) n \\
n
\end{array}\right) .
$$

\section{Acknowledgements}

The ideas in this paper originated as the author's solution [8] to a problem on the mathematics question-and-answer site Mathematics Stack Exchange.

\section{References}

[1] Ora Engelberg. On some problems concerning a restricted random walk. Journal of Applied Probability, 2:396-404, 1965.

[2] William Feller. An Introduction to Probability Theory and Its Applications, volume I. John Wiley \& Sons, New York, second edition, 1957.

[3] I.P. Goulden and Luis G. Serrano. Maintaining the spirit of the reflection principle when the boundary has arbitrary integer slope. Journal of Combinatorial Theory, Series A, 104:317-326, 2003.

[4] Katherine Humphreys. A history and survey of lattice path enumeration. Journal of Statistical Planning and Inference, 140:2237-2254, 2010.

[5] J. R. McGregor, T. V. Narayana, and Z. M. Özsoyoglu. On touchings, crossings, and meetings of lattice paths with the diagonal. Utilitas Mathematica, 30:45-51, 1986.

[6] Sri Gopal Mohanty. Lattice Path Counting and Applications. Academic Press, New York, 1979.

[7] Heinrich Niederhausen. How many paths cross at least $\ell$ given lattice points? Congressus Numerantium, 36:161-173, 1982.

[8] Mike Spivey. Combinatorial proof of $\left(\begin{array}{c}3 n \\ n\end{array}\right) \frac{2}{3 n-1}$ as the answer to a coin-flipping problem. http://math.stackexchange.com/q/66146 (version: 2011-11-09). 